\section{Deep vein thrombosis, ecythyma gangrenosum and heparin-induced thrombocytopenia occurring in a man with a heterozygous Factor $\mathbf{V}$ Leiden mutation}

\author{
Mariya Apostolova, Baoying Weng, \\ Harry H. Pote, Harold Ashcraft, \\ Curtis Goldblatt, Paul V. Woolley \\ Department of Medicine, Department \\ of Pathology and Conemaugh Cancer \\ Care Center, Conemaugh Memorial \\ Medical Center, Johnstown, PA, USA
}

\begin{abstract}
Skin necrosis and limb gangrene are occasional thrombotic manifestations of anticoagulation therapy. We report a man heterozygous for the Factor V Leiden (FVL) mutation, and with a history of recurrent deep venous thrombosis, who initially presented with a necrotic skin lesion of the right flank while on warfarin therapy with a therapeutic international normalized ratio. Warfarin was discontinued and he received intravenous heparin. Thereafter he developed thrombocytopenia and pedal erythema and was diagnosed with heparininduced thrombocytopenia (HIT). Heparin was replaced with argatroban. He ultimately underwent bilateral below-knee amputations for the thrombotic complications of the HIT. The initial necrotic lesion healed with antibiotics and wound care. Pathologic examination of multiple biopsy specimens revealed two separate lesions. One was necrotic tissue infiltrated with methicillin resistant Staphylococcus aureus having features of ecthyma gangrenosum. The second showed thrombotic changes consistent with HIT. The case illustrates the differential diagnosis of skin necrosis and limb gangrene in patients on warfarin and heparin, and also the clinical complexities that can occur in a FVL heterozygote.
\end{abstract}

\section{Introduction}

The Factor V Leiden (FVL) mutation slows degradation of factor $V$ and predisposes to clinical hypercoagulability, usually resulting in deep venous thrombosis (DVT). Heterozygotes for the mutation have a 5-10 fold increase in thrombotic episodes over normal, while homozygotes may have up to a 50-79 fold increase. ${ }^{1,2}$ Risk factors that potentiate thrombus formation include smoking, estrogen-con- taining oral contraceptives, pregnancy, recent surgery and prolonged immobility. The heterozygous condition is not treated unless complications have occurred; rather patients are counseled on risk factors and life style changes to minimize occurrence of thrombotic episodes. Concurrent conditions such as heparin-induced thrombocytopenia (HIT) have been reported with FVL, although it is not clear if a cause and effect relationship exists. ${ }^{3}$ Ecthyma gangrenosum (EG) associated with methicillin resistant Staphylococcus aureus (MRSA) has been infrequently reported. ${ }^{4}$ Here we report a complex case that included EG with tissue infiltration by MRSA and also HIT with thrombotic complications requiring bilateral below knee amputations in a man heterozygous for the FVL (R506Q) mutation.

\section{Case Report}

A 59 year old male presented with a $30 \times 12$ cm discolored lesion of acute onset on his right flank (Figure 1). His history included a twenty pack year smoking history and recurrent DVT of the lower extremities and subsequent arterial thrombosis of the left lower extremity requiring angiography and thrombolysis. He had been on warfarin for five years and his therapeutic international normalized ratio (INR) was 2.6. He had noticed a dark and painful lesion on the skin of his right lower back arise over forty eight hours. The initial impression was that this represented warfarin induced skin necrosis (WISN), but doubt was also raised because the timing did not seem appropriate. A computed tomography (CT) scan demonstrated hemorrhage and edema in the subcutaneous tissues of the right flank and buttock. Warfarin was stopped and intravenous heparin begun, which he had previously received without incident.

He was treated for thrombophlebitis of the right leg with heparin and warfarin in January 2005. He again presented in April 2006 with a pulseless and cyanotic left lower extremity. He had discontinued the warfarin in December 2005 because of medication cost. His prothrombin time (PT) was $10.7 \mathrm{sec}$, INR was 1.0. Arterial catheterization showed extensive thrombotic disease of the left superficial femoral artery and the left popliteal artery. He received intravenous heparin, tissue thromboplastin activator and eptifibatide. Angiography less than twenty four hours later showed only minimal residual arterial thrombus. He was discharged on Lovenox and warfarin, and was cautioned about adherence to his prescribed medications and about smoking cessation.

His admission temperature was $36.4^{\circ} \mathrm{C}$. Routine laboratory values were unremarkable. A 30x12 cm dark red to black skin discoloration
Correspondence: Paul V. Woolley, Conemaugh Cancer Care Center, 1020 Franklin St Johnstown PA 15905, USA.

Tel. + 1.814.5349165.

E-mail: woolleypv@atlanticbb.net

Key words: Factor V Leiden, ecthyma gangrenosum, heparin induced thrombocytopenia, deep vein thrombosis.

Conflict of interests: the authors declare no conflict of interests.

Contributions: MA, BW, CG, PVW, data gathering, drafting the manuscript and revising it critically; HHP, HA, data gathering, and revising the manuscript critically.

Received for publication: 11 August 2012.

Revision received: 23 September 2012.

Accepted for publication: 11 0ctober 2012.

This work is licensed under a Creative Commons Attribution NonCommercial 3.0 License (CC BYNC 3.0).

(C) Copyright M. Apostolova et al., 2012

Licensee PAGEPress, Italy

Hematology Reports 2012; 4:e20

doi:10.4081/hr.2012.e20

on the right flank extended upward from the pelvic brim (Figure 1). PT was $26.5 \mathrm{sec}$, INR 2.6 and partial thromboplastin time (PTT) 29 sec. Liver function tests showed elevated alkaline phosphatase at $168 \mathrm{IU} / \mathrm{L}$ and GGTP of $140 \mathrm{IU} / \mathrm{L}$. Doppler studies of the lower extremities showed no acute DVT. Chest CT showed only scattered bilateral pleural thickening. A hypercoagulable panel, drawn after warfarin had been discontinued, showed protein S 84\% and protein $\mathrm{C} 86 \%$. The sole abnormality was a single copy of the FVL (R506Q) mutation. Anticardiolipin antibodies were not present, cryoglobulin levels were negative and multiple fibrinogen determinations and PTTs were normal. Serum cholesterol was $164 \mathrm{mg} / \mathrm{dL}$. Four days after presentation he underwent debridement of necrotic flank tissue and placement of a wound vacuum assisted closure. At that time, he was febrile and vesicles with hemorrhagic fluid had developed in the area of the tissue necrosis. The size of the nonviable tissue was $27 \times 12 \mathrm{~cm}$. Blood cultures showed no growth. Biopsy of the right flank lesion showed changes consistent with EG (see pathology section). This patient had received heparin on previous occasions without incident, but sixteen days after admission and after initiation of heparin he experienced a very typical onset of HIT. Bluish purplish discoloration involving the first three toes of the right foot and extending into the ankle region developed overnight; the area was tender to palpation and the foot 
was cool to touch. Simultaneously his platelet count had fallen from 496 thou/ $/ \mathrm{mm}^{3}$ to 156 thou $/ \mathrm{mm}^{3}$ over the preceding four days. HIT was immediately suspected, heparin was discontinued and argatroban was begun. No acute changes of the left lower extremity were observed. Arterial Doppler study showed severe small vessel occlusive disease. A dorsalis pedis pulse was detected on the Doppler examination but was not palpable. Lower extremity angiography revealed right anterior tibial artery occlusion. His left foot subsequently developed gangrene also. Antibodies to heparin/platelet factor 4 (H/PF4) complexes were present. Seventeen days later drainage cultures showed rare colonies of MRSA and vancomycin was begun. The lower extremity lesions did not improve (Figure 2). He then developed a new lesion on his left lower abdominal wall and left groin, similar in appearance to the original presenting lesion (Figure 3). He was started on steroids for possible necrotizing vasculitis. His legs worsened in color and extension of the blue discoloration and pain occurred. C3 and C4 complement levels were normal at 177 and $29 \mathrm{mg} / \mathrm{dL}$ respectively. ANCA panel for vasculitis, hepatitis panel and anti-nuclear antibody screen were non-contributory. He was felt to have arterial and venous thrombosis secondary to HIT. His legs were not salvageable and he underwent bilateral amputations at Mercy Hospital Pittsburgh, USA. The lesions on his back and anterior abdomen healed with antibiotic treatment, surgical debridement and wound care. Review of the pathology at Conemaugh Memorial Medical Center, Mayo Clinic and Mercy Hospital Pittsburgh agreed that two separate lesions, namely EG and thrombosis secondary to HIT, were present.

\section{Pathology}

Biopsy tissue was examined from the initial right flank lesion (Figure 1) and from the amputated limbs (Figure 2). The lesion on the back was confluent with a similar lesion on the anterior abdomen and this is the area that was biopsied (Figure 3). The initial lesion showed necrotizing vasculitis with associated epidermal necrosis and hemorrhage (Figures 4 and 5). Numerous extravasated red blood cells were seen with focal areas of subepidermal bullous eruption. Venous thrombosis, but not arterial thrombosis was present (Figure 5). The tissue was infiltrated with MRSA, identified with a MRSA-specific monoclonal antibody from Santa Cruz Biotechnology Inc. [MRSA (NYR MRSA16): sc-73327] (Figures 6 and 7). The bacteria were identified both within blood vessel lumina and soft tissue. There was a mild acute inflammatory cell infiltrate around the small and medium sized blood vessels in the dermis and the subcutaneous fat with associated endothelial cell damage. CD61 immunostain highlighted venous intravascular platelet microthrombus formation (Figure 8). CD61 is an epitope of the b-3 integrin chain present on platelets, and its immunostain is more sensitive than histological evaluation for the detection of microthrombi. ${ }^{5}$ Brown and Brenn (B\&B) stain showed Gram-positive cocci within the media of vessel walls and inside the vascular lumina. Gomori methenamine silver (GMS) and periodic acid Schiff (PAS) stains were negative for fungal organisms. These findings of hemorrhagic epidermal necrosis, acute inflammatory infiltration, bacteria in the blood vessels, vasculitis and thrombosis were considered diagnostic of EG.

Tissue from the amputated lower extremities displayed a different pattern, with thrombosis involving arterioles and venules, necrosis associated with deep cellulitis, and intravascular thrombus formation in both skin and subcutis (Figure 9) as demonstrated by CD61 immunostain (Figures 10 and 11). Thrombus was present in the anterior tibial artery. GMS, PAS and B\&B stains were negative for bacterial or fungal organisms.

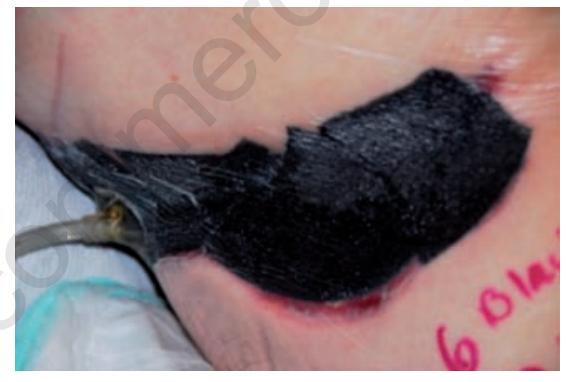

Figure 1. The figure depicts the initial right flank lesion after wound VAC placement.

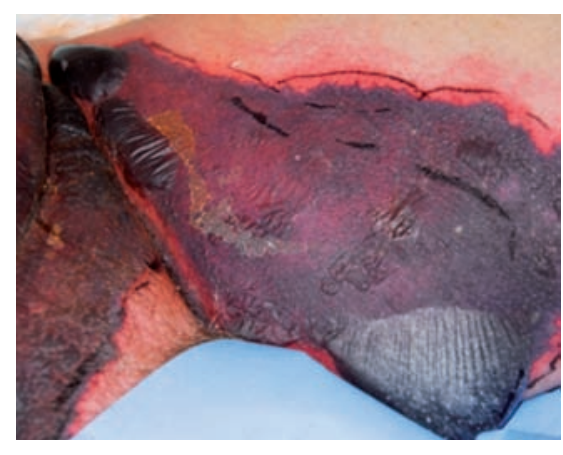

Figure 3. Gross view of left abdominal wall and groin area lesion.

\section{Discussion}

The heterozygous FVL mutation ranges in clinical presentation from benign to severe thrombosis. When thrombotic events occur, the duration of anticoagulation depends upon the severity and the repetitive nature of the event. This patient was a candidate for lifetime anticoagulation because of his history of two episodes of DVT while on anticoagulation and a third episode of mixed arterial and venous thrombosis that occurred while he was off of warfarin. It is probable that the initial two episodes of DVT were secondary to his FVL status, but the third one was due to the absence of warfarin therapy because of his medication noncompliance.

An important issue was to distinguish WISN from other possible entities. The pathologic features of the skin lesion that prompted the diagnosis of EG included necrotizing vasculitis and the presence of MRSA in the biopsies. Table 1 lists other conditions that were considered. He did not have disseminated intravascular coagulation, since his fibrinogen and PTT

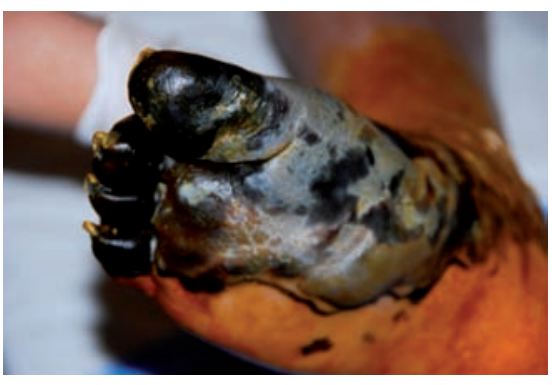

Figure 2. Lower extremity gangrene secondary to both arterial and venous thrombosis.

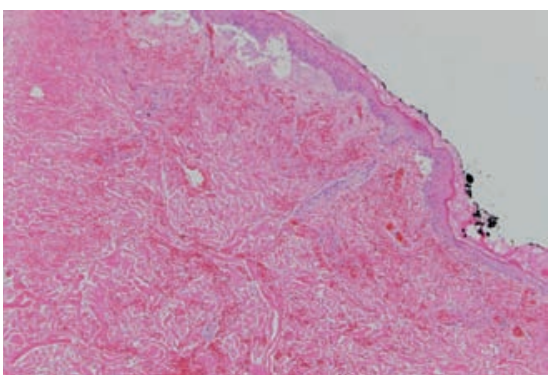

Figure 4. Epidermal necrosis with sub epidermal bullous formation and extravasated red blood cells (H\&E stain, $100 \times$ magnification) 
were normal on multiple occasions. There was no supporting evidence for any of the others on either clinical or pathologic grounds.

Warfarin acts on the procoagulant factors II, VII, IX and X and on the anticoagulant factors protein $\mathrm{S}$ and protein $\mathrm{C}$. WISN depends on an asynchronous fall in the procoagulant and anticoagulant portions of the coagulation cascade following warfarin initiation. Protein C has a shorter half-life (6 hours) than the procoagulant factors and its rapid clearance leads to a transient hypercoagulable state. ${ }^{6}$ Skin necrosis typically occurs within 10 days after starting warfarin therapy. ${ }^{6,7}$ It involves the thighs, buttocks and other areas of the body containing increased areas subcutaneous tissue, ${ }^{6,7}$ producing pain and erythema. The redness evolves into a dark red to black discoloration, leading progressively to gangrene, sometimes with blistering. ${ }^{6}$ The histopathological findings are diffuse microthrombi within dermal and subcutaneous capillaries, venules and deep veins, with endothelial cell damage resulting in ischemic skin necrosis and red blood cell extravasation. ${ }^{6}$ Vascular inflammation and arterial involvement are not recog- nized features of WISN; this facilitates its differentiation from other vascular processes. ${ }^{7}$ Warfarin necrosis has also been reported in patients receiving heparin. ${ }^{8-10}$ Later occurrence up to 18 months may be associated with discontinuation and re-initiation of warfarin or displacement of albumin-bound warfarin by other drugs. ${ }^{11}$ However, this patient had been on warfarin therapy for years and neither of these factors seemed to apply.

Two subtypes of HIT exist. Type I is likely non-immune. ${ }^{12,13}$ It occurs within five days of heparin therapy, the thrombocytopenia is mild and there is no associated risk of thrombosis. ${ }^{12,13}$ Type II occurs when an antibody reacts with a complex formed between PF 4 and heparin. ${ }^{12-14}$ producing $\mathrm{PF} 4 / \mathrm{H} / \mathrm{IgG}$ complexes that bind to platelet Fc receptors. Activation of platelets and intrinsic tissue factor and increased thrombin generation result, leading to a hypercoagulable state and thrombosis. ${ }^{13}$ The acute arterial or venous occlusion occurs because of intravascular platelet aggregation. Cutaneous necrosis can occur, usually 6 to 13 days after onset of therapy. Several reports describe simultaneous FVL mutation and HIT and also the concomitant existence of WISN and HIT. ${ }^{8-10,15}$ Some authors speculate that FVL mutations increase the rate at which thrombocytopenia and thrombosis develop in patients with HIT. ${ }^{14}$ Warfarin treatment of HIT patients can lead to severe thrombotic complications, including limb gangrene.

EG was originally described in association with Pseudomonas aeruginosa septicemia, ${ }^{16-20}$ but later reports include organisms such as Staphylococcus aureus, which is occasionally MRSA, group A Streptococcus, Aeromonas hydrophila, Serratia marcescens and Pseudomonas maltophilia. ${ }^{16-19}$ EG occurs as a Type I lesion associated with septicemia, and a Type II lesion associated only with local bacterial colonization. ${ }^{16,19}$ In this case, MRSA was identified by monoclonal antibody staining of tissue biopsies but not on blood cultures, placing it in the Type II category. In either case pathologic progression occurs as bacterial invasion produces inflammation and thrombosis of post-capillary vessels. Arterial involvement, either from bacterial invasion or venous obliteration, causes separation of dermis and epidermis and subsequent bullae formation.

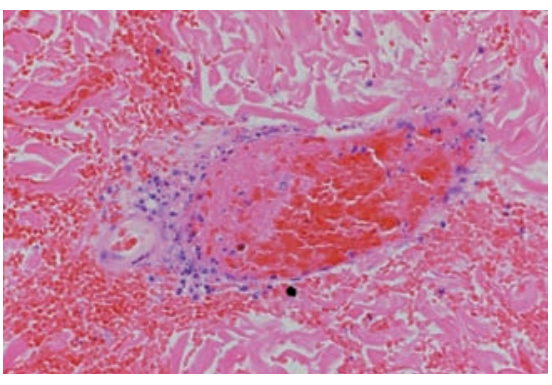

Figure 5. Dermal venous thrombus with acute inflammatory cell infiltration and endothelial cell damage (H\&E stain, 400 $\times$ magnification).

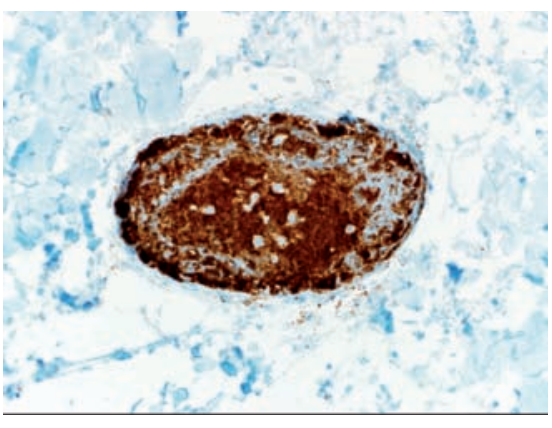

Figure 8. CD61 immunostain from the original lesion shows venous platelet microthrombus (Immunohistochemical (IHC) stain for CD61, 400 $\times$ magnification).

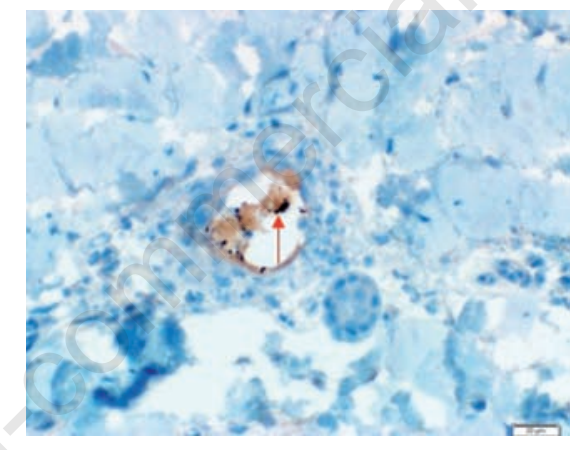

Figure 6. MRSA specific antibody stain demonstrating bacteria in a blood vessel.

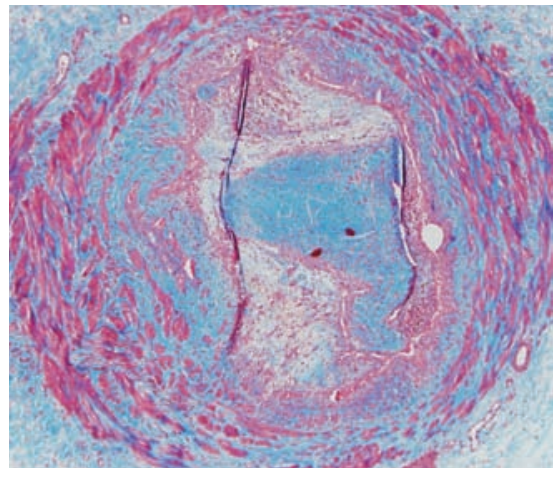

Figure 9. Thrombus in anterior tibial artery (Trichrome stain, 100 $\times$ magnification).

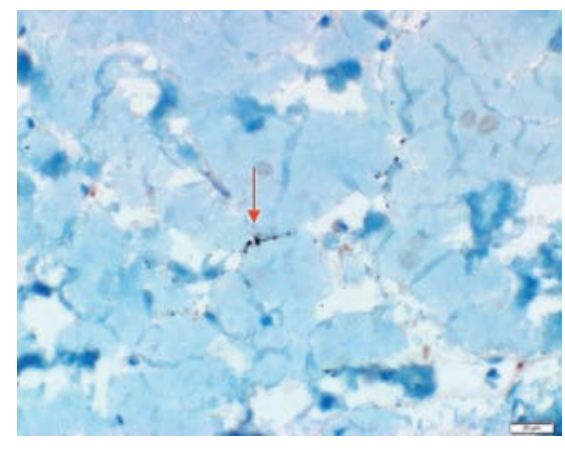

Figure 7. MRSA antibody stain demonstrating bacteria in soft tissue.

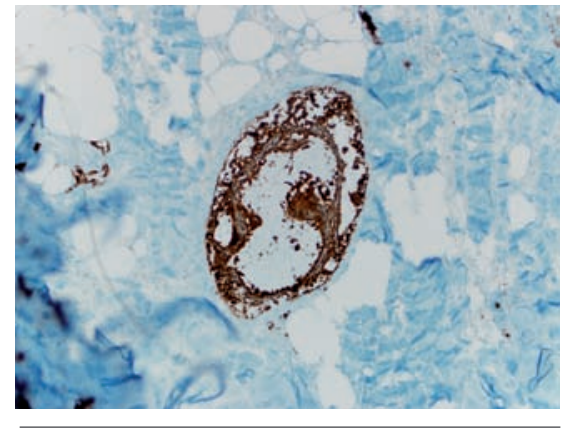

Figure 10. CD61 immunostain of the lower extremity lesion shows platelet microthrombus (IHC stain for CD61, $250 \times$ magnification). 


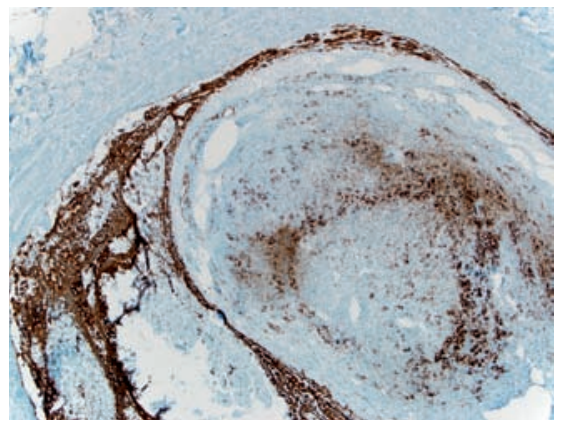

Figure 11. CD61stain shows platelet thrombus (IHC stain for CD6, 100 $\times$ magnification).

Table 1. Differential diagnosis of warfatin induced skin necrosis.

Differential diagnosis of warfarin induced skin necrosis

Acute necrotizing fasciitis

Calciphylaxis

Cryoglobulinemia (Types II and III)

Decubitus ulcer

Disseminated intravascular coagulopathy

Purpura fulminans

Ecthyma gangrenosum

Fournier's gangrene

Hematoma

Heparin-induced antiplatelet antibodies

Inflammatory breast cancer

Purple toe cholesterol embolism syndrome

Pyoderma gangrenosum

Hosp Phys 1999;35:55-7.

The bacterial load of the bullae increases, producing a central necrotic area surrounded by an erythematous halo. No discharge is present. The bulla sloughs, exposing an ulcerated area. These lesions can occur anywhere on the body, commonly the groin, axilla and anogenital region. ${ }^{19}$ This process can occur rapidly, often within 24 hours. ${ }^{18}$ Bacteria are numerous in the media and adventitia of the necrotic vessels, but typically spare the intima. ${ }^{9}$ Our case differs from other descriptions in that bacteria were found in blood vessel lumina and in soft tissue rather than in the media and adventitia. There was also an absence of tissue inflammatory reaction. Despite these differences the overall assessment of the pathology was that it represented EG. The original source of the MRSA invasion was unclear. In conclusion, this case illustrates a complex set of severe thrombotic complications in a FVL heterozygote. A necrotic lesion in a patient on warfarin may appear to be WISN, but the differential diagnosis extends further.

\section{References}

1. Lovejoy AE, Bush RA, Pong AL, Hilfiker ML. A male adolescent with left iliac thrombophlebitis and heterozygosity for factor $\mathrm{V}$ Leiden mutation. J Pediatr Surg 2009;44: 1640-2.

2. Johnson JD. Factor V Leiden mutation.
3. Galindo M, Gonzalo E, Martinez-Vidal MP, et al. Immunohistochemical detection of intravascular platelet microthrombi in patients with lupus nephritis and antiphospholipid antibodies. Rheumatology (Oxford) 2009;48:1003-7.

4. Mandegar MH, Saidi B, Roshanali F. Extensive arterial thrombosis in a patient with factor $\mathrm{V}$ Leiden mutation. Interact Cardiovasc Thorac Surg 2010;11:127-9.

5. Bontempo FA, Hassett AC, Faruki H, et al. The factor $V$ Leiden mutation: spectrum of thrombotic events and laboratory evaluation. J Vasc Surg 1997;25:271-5.

6. McKnight JT, Maxwell AJ, Anderson RL. Warfarin necrosis. Arch Fam Med 1992; 1:105-8.

7. Nazarian RM, Van Cott EM, Zembowicz A, Duncan LM. Warfarin-induced skin necrosis. J Am Acad Dermatol 2009;61:325-32.

8. Abdel-Wahab OI, Rosovsky RP, Warth JA. Warfarin-induced skin necrosis in a patient with heparin-induced thrombocytopenia: two diseases or one? Acta Haematol 2008;120:117-22.

9. Howard-Thompson A, Usery JB, Lobo BL, Finch CK. Heparin-induced thrombocytopenia complicated by warfarin-induced skin necrosis. Am J Health Syst Pharm 2008;65:1144-7.

10. Kaiber FL, Malucelli T0, Baroni Edo R, et al. Heparin-induced thrombocytopenia and warfarin-induced skin necrosis: case report. An Bras Dermatol 2010;85:915-8.

11. Essex DW, Wynn SS, Jin DK. Late-onset warfarin-induced skin necrosis: case report and review of the literature. Am J Hematol 1998;57:233-7.

12. Chong BH. Heparin-induced thrombocytopenia. J Thromb Haemost 2003;1:1471-8.

13. Franchini M. Heparin-induced thrombocytopenia: an update. Thromb J 2005;3:14.

14. Warkentin TE. Think of HIT. Hematol Am Soc Hematol Educ Program 2006:408-14.

15. Chaubey S, Davidson SJ, Desouza AC. Heparin induced thrombocytopenia in a patient with factor $\mathrm{V}$ Leiden following cardiac surgery. Interact Cardiovasc Thorac Surg 2009;9:1023-5.

16. Sen H, Inangil G, Sahin L, et al. Ecthymagangrenosum-like lesions associated with methicillin-resistant Staphylococcus aureus infection. Int $\mathrm{J}$ Infect Dis 2009;13: e173-5.

17. Gençer S, Ozer S, Ege Gül A, et al. Ecthyma gangrenosum without bacteremia in a previously healthy man: a case report. J Med Case Rep 2008;2:14.

18. Khan F, Saul T. Ecthyma gangrenosum. J Emerg Med 2012;43:e133-4.

19. Patel JK, Perez OA, Viera MH, et al. Ecthyma gangrenosum caused by Escherichia coli bacteremia: a case report and review of the literature. Cutis 2009; 84:261-7.

20. Singh N, Devi M, Devi S. Ecthyma gangrenosum: a rare cutaneous manifestation caused by Pseudomonas aeruginosa without bacteremia in a leukemic patient. Indian J Dermatol Venereol Leprol 2005; 71:128-9. 